\title{
Simulation of Local Stability Analysis of An Epidemic Model with Constant Removal Rate of the Infective Between 2017-2019
}

\author{
Tadesse Lamessa Edae Sisay Tadese \\ College of Natural and Computational science, Department of Applied Mathematics Wolita sodo university, PO \\ box 138, Wolaita Sodo, Ethiopia
}

\begin{abstract}
In this thesis we consider an epidemic model with a constant removal rate of infective individuals is proposed to understand the effect of limited resources for treatment of infective on the disease spread. It is found that it is unnecessary to take such a large treatment capacity that endemic equilibria disappear to eradicate the disease. It is shown that the outcome of disease spread may depend on the position of the initial states for certain range of parameters. It is also shown that the model undergoes a sequence of bifurcations including saddle-node bifurcation, subcritical Hopf bifurcation.
\end{abstract}

Keywords: Epidemic model, nonlinear incidence rate, basic reproduction number, local and global stability DOI: $10.7176 / \mathrm{JHMN} / 61-01$

Publication date: April $30^{\text {th }} 2019$

\section{INTRODUCTION}

Mathematical epidemiology contributed to the understanding of the behavior of infectious diseases, its impacts and possible future predictions about its spreading. Mathematical models are used in comparing, planning, implementing, evaluating and optimizing various detection, prevention, therapy and control programs. The asymptotic behavior of epidemic models has been studied by many researchers [1, 4-6,8-11]. Periodic oscillations have been observed in the incidence of many infectious diseases, including measles, mumps, rubella, chickenpox, and influenza.

In this thesis, we suppose that the capacity for the treatment of a disease in a community is a constant $r$. In order to easily understand its effect, we consider a case that the removal rate of infective equals $r$. This means that we use the maximal treatment capacity to cure or isolate infective so that the disease is eradicated. This can occur if the disease is so dangerous that we hope to wipe out it quickly, or the disease spreads rapidly so that the treatment capacity is insufficient for treatment in a period (flu, for example).

\subsection{Methodology}

Mathematical modeling and Numerical simulation are used in this thesis. We considered the SIR model to study the dynamics of the infectious disease. The effect of main facts was simulated using numerical method.

\subsection{MATHEMATICAL MODEL}

In this section, we present a mathematical model which describes the dynamics of infectious disease. To formulate the model we consider that the total population is constant, and that a small number of infected individuals are introduced into a large population.

Let $\mathrm{S}(\mathrm{t}), \mathrm{I}(\mathrm{t})$ and $\mathrm{R}(\mathrm{t})$ represent the number of susceptible, infective, and recovered individuals at any time $\mathrm{t}$ respectively. If we think about the process of a disease transmission that it's the SIR framework we have a flow of individuals from the susceptible group to the infected group and then to the recovered group.

\subsection{The Assumptions and Notation}

The following assumptions are used to construct the SIR model:

1. The population has a constant size $\mathrm{N}$, the natural death rate of the population is denoted by $\mu$, the natural recovery rate of the infective individuals is represented by $\gamma$, the recruitment rate of the population is denoted by $\mathrm{A}$ and the transmission rate is denoted $\beta$

2. The number of infected increases at a rate proportional to both the number of infected and the susceptible: $\beta$ SI with $\beta>0$. And the number of the susceptible decreases at this same rate.

3. The rate of removal of infected to the $R$ class is proportional to the number of infected only: $\gamma I$, with $\gamma>$ 0 . And the number of removed increases at this same rate.

4. The incubation time is negligible, so that a susceptible that catches the disease becomes infectious immediately.

Assuming the disease spreads into a population that is totally susceptible, the susceptible individuals have never come into contact with the disease and are able to capture the disease, after which they move into the infectious class. Infectious individuals spread the disease to the susceptible, and remain in the infectious class for a given period of time (the infectious period) before moving into the recovered/removed class. 
If we assume that individuals are mixed randomly then each potential transmission may be from an infected population to a susceptible population which results in a new infected population. Or a transmission may occur from an infected population to another infected population in which case nothing happening since the person is already infected. Or the potential transmission may occur from an infected person to a recovered or immune person. In this case again nothing changes. This description of the SIR model was made more mathematical by a formulated differential equation for the proportion of individuals in each class.

\subsection{The Model Equation}

Based on the above assumption the dynamics of the disease can be express using the system of ordinary differential equation

$$
\begin{aligned}
& \frac{d s}{d t}=A-\mu S-\beta S I, \\
& \frac{d I}{d t}=\beta S I-(\mu+\gamma) I-h(I), \\
& \frac{d R}{d t}=\gamma I+h(I)-\mu R
\end{aligned}
$$

where all parameter are positive and $\mathrm{h}(\mathrm{I})$ is the removal rate of infective individuals due to the treatment of infective. The definition of $\mathrm{h}(\mathrm{I})$ implies that a constant removal rate for the infective is used until the disease disappears. We suppose that the treated infective become recovered when they are treated in treatment sites. Suppose that

$$
h(I)=\left\{\begin{array}{lll}
r & \text { for } & I>0 \\
0 & \text { for } & I=0
\end{array}\right.
$$

where $r>0$ is a constant and it represents the capacity of treatment for infective. This means that we use a constant removal rate for the infective until the disease disappears.

\begin{tabular}{|c|l|}
\hline Parameters & \multicolumn{1}{|c|}{ Parameters definition } \\
\hline $\mathrm{A}$ & Recruitment rate(New born or immigrants) \\
\hline $\mathrm{M}$ & Natural death rate \\
\hline $\mathrm{B}$ & $\begin{array}{l}\text { The contact rate, defined to be the average number of effective contacts with } \\
\text { other(susceptible) individuals per infective per unit time }\end{array}$ \\
\hline$\Gamma$ & The rate at which an infectious individual recovered per unit time \\
\hline
\end{tabular}

Table 2.1 Parameters and their definitions

\begin{tabular}{|c|l|}
\hline Variables & \multicolumn{1}{c|}{ Definition } \\
\hline $\mathrm{S}(\mathrm{t})$ & The number of susceptible individuals at time, $\mathrm{t}$ \\
\hline $\mathrm{I}(\mathrm{t})$ & The number of infected individuals at time, $\mathrm{t}$ \\
\hline $\mathrm{R}(\mathrm{t})$ & The number of recovered individuals at time, $\mathrm{t}$ \\
\hline
\end{tabular}

Table 2.2 Variables and definitions of populations used as variable

\section{MODEL ANALYSIS}

This section deals with the study of stability properties of different equilibriums point of the model. Stability analysis is crucial in this study since we would be able to know whether disease free equilibrium point and endemic equilibrium point would be stable so that the disease would persist or not.

\subsection{Positivity and Boundedness of Solutions}

We can show from system (1) that the state variables are non-negative and the solutions remain positive for all time $t \geq 0$. Here the parameters in the system are assumed to be positive. We also show that the feasible solutions are bounded in a region:

$$
\left.\Gamma=\left\{(S, I, R) \in R^{3}: S+I+R \leq \frac{A}{\mu}: \text { where } S \geq 0, I \geq 0, R \geq 0\right)\right\}
$$

Lemma1 : [25] Let the initial values $\{S(0) \geq 0, I(0) \geq 0, R(0) \geq 0\}$ be in $\Gamma$ for $t \geq 0$ then the solution set $\{S(t), I(t)$, $R(t)\}$ is positive for all $t \geq 0$. 
Proof: Assume that the initial values $\{S(0) \geq 0, I(0) \geq 0, R(0) \geq 0\}$ be in $\Gamma$ for $t \geq 0$. From the first equation we have

$$
\frac{d s}{d t}=A-\mu S-\beta S I \geq-(\mu+\beta I) S
$$

Therefore,

$$
\frac{d s}{d t} \geq-(\mu+\beta I) S
$$

$\frac{d S}{S} \geq-(\mu+\beta I) d t$, I is a function of $\mathrm{t}$

Integrating both sides of the inequalities, we get

Solving for $\mathrm{S}(\mathrm{t})$ we get

$$
\begin{gathered}
\ln \left(S(t) \geq-\int(\mu+\beta I) d t\right. \\
S(t) \geq e^{-\int(\mu+\beta I) d t}+C
\end{gathered}
$$

But at $\mathrm{t}=0$, we have

$$
S(t)=C_{0} e^{-(\mu+\beta I) t} .
$$

Hence, $S(t) \geq 0$.

$$
S(t) \geq S(0) e^{-(\mu+\beta) t}, \text { since }(\mu+\beta I)>0
$$

From the second equation of system (1) we have

$$
\frac{d I}{d t}=\beta S I-(\mu+\gamma) I-r \geq-(\mu+\gamma) I
$$

Integrating both sides of the inequalities, we get

Then

$$
\ln (I(t)) \geq-(\mu+\gamma) t+C .
$$

But at $\mathrm{t}=0$, we have

$$
\mathrm{I}(\mathrm{t}) \geq \mathrm{C}_{0} \mathrm{e}^{-(\mu+\gamma) \mathrm{t}} .
$$

$$
\mathrm{I}(\mathrm{t}) \geq \mathrm{I}(0) \mathrm{e}^{-(\mu+\gamma) \mathrm{t}} \geq 0, \text { since }(\mu+\gamma)>0
$$

Hence, $\mathrm{I}(\mathrm{t}) \geq 0$.

From last equation in system (1) we obtained

which has an integrating factor $\mathrm{I}(\mathrm{t})=\mathrm{e}^{\mu \mathrm{t}}$

$$
\frac{d R}{d t}=\gamma I+h(I)-\mu R \geq \gamma I-\mu R .
$$

Integrating the inequalities above, we obtain

$$
\frac{d R}{d t} e^{\mu t}=\gamma I e^{\mu t}-\mu R(t) e^{\mu t}
$$

$$
R(t)=\frac{\gamma I}{\mu}+C e^{-\mu t},
$$

At $t=0$ we have

$$
R(0)=\frac{\gamma I}{\mu}+C
$$

The solution of the linear differential equation then becomes

Therefore,

$$
R(t)=R(0) e^{-\mu t}+\frac{\gamma I}{\mu}\left(1-e^{-\mu t}\right)
$$

$$
R(t)=R(0) e^{-\mu t}+\frac{\gamma I}{\mu}\left(1-e^{-\mu t}\right) \geq 0,
$$


Hence, all the state variables are positive for all time $\mathrm{t} \geq 0$.

Lemma 2: [25]The solutions for system (1) are contained and remain in the region $\Gamma$ for all time $t \geq 0$

Proof: Suppose that $(S(t), I(t), R(t))$ is a solution of system (1). Then since $N(t)=S(t)+I(t)+R(t)$ is the population at any given time $t$, it is non-negative, that is

$$
\mathrm{N}(\mathrm{t}) \geq 0 \text { for all } \mathrm{t} \geq 0 \text {. }
$$

Thus, the lower bound for $\mathrm{S}(\mathrm{t}), \mathrm{I}(\mathrm{t})$ and $\mathrm{R}(\mathrm{t})$ is 0 . To find the upper bound of the system consider the following equation

Then

$$
\mathrm{N}=\mathrm{S}+\mathrm{I}+\mathrm{R} \text { and } \mathrm{N}^{\prime}=(\mathrm{S}+\mathrm{I}+\mathrm{R})^{\prime}
$$

$$
(\mathrm{S}+\mathrm{I}+\mathrm{R})^{\prime}=\mathrm{A}-\mu \mathrm{S}-\mu \mathrm{I}-\mu \mathrm{R}=\mathrm{A}-\mu(\mathrm{S}+\mathrm{I}+\mathrm{R})=\mathrm{A}-\mu \mathrm{N}
$$

The solution of the linear differential equation then becomes

It follows that

$$
N(t)=N(0) e^{-\mu t}+\frac{A}{\mu}\left(1-e^{-\mu t}\right)
$$

That is $\mathrm{N}$ is bounded.

$$
\lim _{t \rightarrow \infty} N(t) \leq \frac{A}{\mu}
$$

Using this result together with Lemma1, we have that $0 \leq \mathrm{N} \leq \mathrm{A} / \mu$ which implies that $\mathrm{N}$ and all other variable ( $\mathrm{S}$, I and R) are bounded and all the solutions starting in $\Gamma$ stay in $\Gamma$ with respect to the system. Therefore, one can show that the removal rate has significant effects on the dynamics of the system. Since the first two equations in system (1) are independent of the variable R it suffices to consider the following reduced model:

$$
\begin{aligned}
& \frac{d s}{d t}=A-\mu S-\beta S I, \\
& \frac{d I}{d t}=\beta S I-(\mu+\gamma) I-r,
\end{aligned}
$$

It is assumed that all the parameters are positive constants.

\subsection{Equilibria and Stability Analysis}

In this section we investigate the existence for the system described by equation. System (3) has always a disease-free equilibrium point and unique endemic equilibrium point. The equilibrium points of the system are obtained by solving the following equations simultaneously

$$
\begin{aligned}
& A-\mu S-\beta S I=0 \\
& \beta S I-(\mu+\gamma) I-r=0
\end{aligned}
$$

We detail each of these equilibriums points as follows based on equation (4)

\subsection{The Disease Free Equilibrium point}

The equilibrium state in the absence of infection is known as the disease free equilibrium point. The disease free equilibrium point is obtained when $\mathrm{I}=0$. Hence the disease free equilibrium point of system (4) is given by $\mathrm{E}_{0}=$ $\left(\frac{A}{\mu}, 0\right)$.

\subsection{The Endemic Equilibrium point}

The equilibrium state with the presence of infection (i.e. $I \neq 0$ ) is known as endemic equilibrium point or non-zero equilibrium point. The endemic equilibrium point of system (4) is the solution of the system of equations

$$
\begin{aligned}
& A-\mu \mathrm{S}-\beta \mathrm{SI}=0 \\
& \quad \beta S I-(\mu+\gamma) \mathrm{I}-\mathrm{r}=0 .
\end{aligned}
$$

Therefore, the endemic equilibrium point is given by

$$
S=\frac{A}{\mu+\beta I}
$$




$$
\beta(\mu+\gamma) I^{2}-(\beta A-\mu(\mu+\gamma)-\beta r) I+r \mu=0
$$

Since the discriminate (form the second equation)

is non-negative, then at least a positive solution exists.

$$
(\beta(A-r)-\mu(\mu+\gamma))^{2}-4 \beta r \mu(\mu+\gamma)
$$

\subsection{The Basic Reproduction Number $\left(\mathbf{R}_{0}\right)$}

The basic reproduction number of an infectious disease is one of the fundamental concepts in mathematical epidemiology. It is defined as the average number of secondary infections caused by an infectious individual during his or her entire period of infectiousness. The basic reproduction number is an important non-dimensional quantity in epidemiology as it sets the threshold in the study of a disease both for predicting its outbreak and for evaluating its control strategies. The condition $\mathrm{R}_{0}<1$ means that every infectious individual will cause less than one secondary infection and hence the disease will die out and when $\mathrm{R}_{0}>1$ every infectious individual will cause more than one secondary infection and hence the disease will invade the population. A large value of $\mathrm{R}_{0}$ may indicate the possibility of a major epidemic. The properties and complexities of $\mathrm{R}_{0}$ depend on the number of infective and intervention strategies.

Thus, the basic reproduction number $\mathrm{R}_{0}$ often takes as the threshold quantity that determines whether or not an infectious disease will spread through a population. Since $S=\frac{A}{\mu}$ for $\mathrm{I}=0, \mathrm{R}_{0}$ is the product of the number A

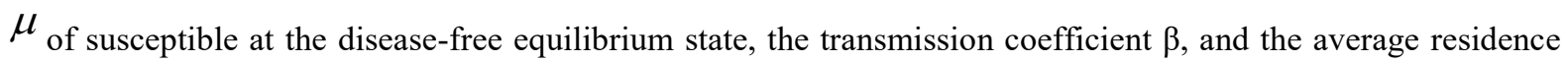

$\frac{1}{\mu+\gamma}$ in the infectious individuals class. Therefore, the basic
\[ R_{0}=\frac{\beta A}{\mu(\mu+\gamma)} \]

Now to find the endemic Equilibrium we substitute $S=\frac{A}{\mu+\beta I}$ into the second equation of system (4) to obtain the quadratic equation

$$
\beta(\mu+\gamma) I^{2}-(\beta A-\mu(\mu+\gamma)-\beta r) I+r \mu=0
$$

Since the basic reproductive number $\mathrm{R}_{0}$ is given by

equation (5) can be rewritten as

$$
R_{0}=\frac{\beta A}{\mu(\mu+\gamma)}
$$

$$
\begin{aligned}
& \qquad \frac{\beta}{\mu} I^{2}-\left(R_{0}-1-H\right) I+\frac{r}{\mu+\gamma}=0 \\
& \qquad \begin{array}{l}
\text { Where } \\
H(\mu+\gamma)
\end{array}
\end{aligned}
$$

Case1: If $\mathrm{R}_{0} \leq 1$ or $\sqrt{R_{0}}-1<\sqrt{H}$. Then equation (6) has no positive solution.

Case2: If $\mathrm{R}_{0}>1$ and $\left(\sqrt{R_{0}}-1\right)^{2}=H$. Then equation (6) has a unique solution

Case 3: If $\mathrm{R}_{0}>1$ and $\left(\sqrt{R_{0}}-1\right)^{2}>H>0$.Then equation (6) has two positive solutions.

Therefore, system (3) has

1. no equilibrium point if $\mathrm{R}_{0} \leq 1$;

2. one interior equilibrium point if (case 1) is satisfied; 
3. two interior equilibrium points if condition (case 3 ) is satisfied. The two endemic equilibrium points of system (3) are given by

Where

$$
\mathrm{E}_{1}=\left(\mathrm{S}_{1}, \mathrm{I}_{1}\right) \text { and } \mathrm{E}_{2}=\left(\mathrm{S}_{2}, \mathrm{I}_{2}\right)
$$

$$
\begin{array}{ll}
I_{1}=\frac{\mu}{2 \beta}\left(\mathrm{R}_{0}-1-H-\sqrt{\left(R_{0}-1-H\right)^{2}-4 H}\right) & S_{1}=\frac{A}{\mu+\beta I_{1}} . \\
I_{2}=\frac{\mu}{2 \beta}\left(\mathrm{R}_{0}-1-H+\sqrt{\left(R_{0}-1-H\right)^{2}-4 H}\right) & S_{2}=\frac{A}{\mu+\beta I_{2}} .
\end{array}
$$

We know that quarantine is an important method to decrease the spread of disease. In classical epidemic models, the treatment rate is assumed to be proportional to the number of infective. In fact, this assumption is irrational because every community should have a suitable capacity for treatment. If it is too large, the community pays for unnecessary cost. If it is too small, the community has the risk of the outbreak of a disease. This means that unnecessary to increase removal rate $r$ to make the disease disappear. The removal rate can be small, so the resources for treatment are saved.

If $\mathrm{N}_{1}=\mathrm{S}+\mathrm{I}$ we have

$$
\frac{d N_{1}}{d t}=\mathrm{A}-\mathrm{r}-\gamma \mathrm{I}-\mu \mathrm{N}_{1} \leq \mathrm{A}-\mathrm{r}-\mu \mathrm{N}_{1} .
$$

Therefore, the positive solution of system (3) is bounded. Note that the non-negative I-axis repels positive solutions of system (3) and that there is no equilibrium on the non-negative S-axis.

If $\mathrm{R}_{0} \leq 1$ or condition (case 1 ) holds, the susceptible population may be any constant in

$[0, \infty)$, which depends on initial conditions. However, the infective go to extinction in a finite time, implying the disappearance of disease.

Theorem 1: [25] The equilibrium point $E_{1}=\left(S_{1}, I_{1}\right)$ is saddle whenever it exists and $E_{2}=\left(S_{2}, I_{2}\right)$ is a center.

Proof: We begin by analyzing the stability of these two equilibria. The Jacobean matrix of (3) is given by

$$
J=\left(\begin{array}{ll}
\frac{\partial S^{\prime}}{\partial S} & \frac{\partial S^{\prime}}{\partial I} \\
\frac{\partial I^{\prime}}{\partial S} & \frac{\partial I^{\prime}}{\partial I}
\end{array}\right)
$$

Now at $\left(\mathrm{S}_{1}, \mathrm{I}_{1}\right)$, the Jacobean matrix becomes

$$
J_{1}=\left(\begin{array}{cc}
-\mu-\beta I_{1} & -\beta S_{1} \\
\beta I_{1} & \beta S_{1}-\mu-\gamma
\end{array}\right)
$$

Note that from the system of equations (3) we have

Then we have

$$
\mathrm{A}-\mu \mathrm{S}_{1}=\beta \mathrm{S}_{1} \mathrm{I}_{1}=(\mu+\gamma) \mathrm{I}_{1}+\mathrm{r}
$$

Therefore,

$$
S_{1}=\frac{A-(\mu+\gamma) I_{1}-r}{\mu}
$$

$$
\begin{aligned}
\operatorname{det}\left(J_{1}\right) & =\left(-\mu-\beta I_{1}\right)\left(\beta S_{1}-\mu-\gamma\right)+\beta^{2} S_{1} I_{1} \\
= & -\beta A+2 \beta(\mu+\gamma) I_{1}+r \beta+\mu(\mu+\gamma) \\
= & \mu(\mu+\gamma)\left[-R_{0}+\frac{2 \beta I_{1}}{\mu}+H+1\right]
\end{aligned}
$$




$$
\begin{aligned}
& =\mu(\mu+\gamma)\left[-R_{0}+2 \frac{\beta}{\mu}\left(\frac{\mu}{2 \beta}\left(\mathrm{R}_{0}-1-H-\sqrt{\left(R_{0}-1-H\right)^{2}-4 H}+H+1\right)\right]\right. \\
& =\mu(\mu+\gamma)\left[-R_{0}+R_{0}-\sqrt{\left(R_{0}-1-H\right)^{2}-4 H}\right] \\
& -\mu(\mu+\gamma)\left[\sqrt{\left(R_{0}-1-H\right)^{2}-4 H}\right]<0
\end{aligned}
$$

It follows that $\left(\mathrm{S}_{1}, \mathrm{I}_{1}\right)$ is saddle point. Since this implies that an eigen-value remains real positive and the other real negative for increasing $\mathrm{r}$.

The Jacobean matrix of system (3) at the equilibrium point $\left(\mathrm{S}_{2}, \mathrm{I}_{2}\right)$ is

$$
J \mid\left(S_{2}, I_{2}\right)=\left(\begin{array}{cc}
-\mu-\beta I_{2} & -\beta S_{2} \\
\beta I_{2} & \beta S_{2}-(\mu+\gamma)
\end{array}\right)
$$

By the same argument we obtain that

$$
\operatorname{det}\left(J_{2}\right)=\mu(\mu+\gamma)\left[\sqrt{\left(R_{0}-1-H\right)^{2}-4 H}\right]>0
$$

Thus the equilibrium point $\mathrm{E}_{2}=\left(\mathrm{S}_{2}, \mathrm{I}_{2}\right)$ is a center.

We can immediately conclude that the endemic equilibrium $E_{1}$ with low number of infected individuals is always a saddle, and that the endemic equilibrium $E_{2}$ with high number of infected individuals is a node or focus.

Theorem 2: [27] If case three (9) holds. Then the equilibrium point $\mathrm{E}_{2}$ is stable if either

$$
\beta(\mathrm{A}-\mathrm{r})-3 \mu^{2}-\mu \gamma-2 \mu^{3} / \gamma \leq 0
$$

or

$0<\beta(\mathrm{A}-\mathrm{r})-3 \mu^{2}-\mu \gamma-2 \mu^{3} / \gamma$ and

$$
0<\beta(A-r)+\frac{1}{2}(2 \mu+\gamma)(\mu+\gamma)\left(1-\sqrt{1+\frac{4 \beta A}{(\mu+\gamma)^{2}}}\right)
$$

and unstable otherwise.

Proof: Since we have

$$
S_{2}=\frac{A-(\mu+\gamma) I_{2}-r}{\mu}
$$

$$
\begin{aligned}
\operatorname{tr}\left(J_{2}\right) & =-2 \mu-\beta I_{2}+\beta S_{2}-\gamma \\
& =-2 \mu-\beta I_{2}+\beta\left(\frac{A-(\mu+\gamma) I_{2}-r}{\mu}\right)-\gamma \\
& =\frac{-2 \mu^{2}-\mu \beta I_{2}+\beta A-\beta(\mu+\gamma) I_{2}-\beta r-\mu \gamma}{\mu} \\
& =-\left(\frac{2 \mu \beta+\gamma \beta}{\mu}\right) I_{2}-\frac{2 \mu^{2}-\beta A+\beta r+\gamma \mu}{\mu}
\end{aligned}
$$

Thus the trace is negative if $2 \mu^{2}+\beta(\mathrm{r}-\mathrm{A})+\gamma \mu \geq 0$.

Let $2 \mu^{2}+\beta(\mathrm{r}-\mathrm{A})+\gamma \mu<0$.

Now we find the condition under which the $\operatorname{tr}\left(\mathrm{J}_{2}\right)=0$. 
Let

$$
D_{1}=-\frac{\mu(\mu+\gamma)}{\beta(2 \mu+\gamma)}\left(\frac{\mu}{\mu+\gamma}+1-R_{0}+H\right) .
$$

From condition (13) we have that $\operatorname{tr}\left(\mathrm{J}_{2}\right)=0$ is equivalent to

$$
I_{2}=-\frac{2 \mu^{2}-\beta A+\beta r+\gamma \mu}{\beta(2 \mu+\gamma)}=D_{1}
$$

$$
\text { If } D_{2}=-\frac{2 \mu}{2 \mu+\gamma}+\frac{\gamma}{2 \mu+\gamma}\left(R_{0}-1-H\right)
$$

It follows from the definition of $\mathrm{I}_{2}$ that $\operatorname{tr}\left(\mathrm{J}_{2}\right)=0$ is equivalent to

$$
D_{2}=\sqrt{\left(R_{0}-1-H\right)^{2}-4 H}
$$

Thus, $\operatorname{tr}\left(\mathrm{J}_{2}\right)$ is negative if

$$
\begin{aligned}
& H \geq R_{0}-1-\frac{2 \mu}{\gamma} \\
& \qquad H \leq R_{0}-1-\frac{2 \mu}{\gamma}
\end{aligned}
$$

Taking squares on both sides of (15) and simplifying the resulting equation, we obtain

$$
D_{3}=r^{2} \beta\left(-3 \gamma \mu-2 \beta A-\gamma^{2}-2 \mu^{2}\right) r+\beta A \mu^{2}=0
$$

Then by using quadratic formula we get

$$
\begin{aligned}
& r=\frac{1}{2 \beta}\left[\left(2 \beta A+(2 \mu+\gamma)(\mu+\gamma) \pm \sqrt{(2 \beta A+(2 \mu+\gamma)(\mu+\gamma))^{2}-4 \beta A\left(\beta A^{2}-2 A \mu^{2}\right.}\right)\right] \\
& =\frac{1}{2 \beta}\left[2 \beta A+(2 \mu+\gamma)(\mu+\gamma) \pm(2 \mu+\gamma)(\mu+\gamma) \sqrt{\frac{(\mu+\gamma)^{2}+4 \beta A}{(\mu+\gamma)^{2}}}\right. \\
& \qquad r=\frac{1}{2 \beta}\left[2 \beta A+(2 \mu+\gamma)(\mu+\gamma)\left(1 \pm \sqrt{\left.\left.1+\frac{4 \beta A}{(\mu+\gamma)^{2}}\right)\right]}\right]\right. \\
& \text { Hence } \quad r=\frac{1}{2 \beta}\left[2 \beta A+(2 \mu+\gamma)(\mu+\gamma)\left(1 \pm \sqrt{1+\frac{4 \beta A}{(\mu+\gamma)^{2}}}\right)\right]
\end{aligned}
$$

Since (16) is equivalent to $\beta A-3 \mu^{2}-\mu \gamma-3 \mu^{3} / \gamma<\beta r$

By definition of $D_{1}$ and $D_{2}$ we have

$$
\underset{\operatorname{Tr}\left(\mathrm{J}_{2}\right)}{=}-\left(\frac{2 \mu \beta+\gamma \beta}{\mu}\right) I_{2}-D_{1}\left(\sqrt{\left(R_{0}-1-H\right)^{2}-4 H}-D_{2}\right)
$$


Thus (16) implies that $\operatorname{tr}\left(\mathrm{J}_{2}\right)<0$. Therefore $\mathrm{E}_{2}$, is stable if condition (19) satisfied.

\subsection{Local Stability Analysis}

\section{Local Stability of Diseases -Free Equilibrium point}

In this section we are going to discuss about the local stability of the disease free equilibrium point. In the absence of the infectious diseases the model has unique diseases free equilibrium at $\mathrm{E}_{0}$. The stability analysis of the diseasefree equilibrium $\mathrm{E}_{0}$ determines the thresholds (reproductive number) of the epidemic. The Jacobean matrix of system (1) at the disease free equilibrium point is given by

$$
\left(\begin{array}{ll}
-\mu & \beta \frac{A}{\mu} \\
0 & \beta \frac{A}{\mu}-\mu-\gamma
\end{array}\right)
$$

Then the characteristic equation of the Jacobean matrix is given by

And hence the corresponding eigen-values are

$$
\lambda^{2}+(2 \mu+\gamma-\beta \mathrm{A} / \mu) \lambda+\mu(\mu+\gamma)-\beta \mathrm{A}=0
$$

$$
\lambda_{1}=-\mu \text { and } \lambda_{2}=-(\mu+\gamma)+\beta \mathrm{A} / \mu \text {. }
$$

In the following theorem established the local stability of the disease free equilibrium point.

Theorem 3: $[27,30]$, The disease free equilibrium $\mathrm{E}_{0}$ is locally asymptotically stable if $\mathrm{R}_{0}<1$, otherwise unstable. Proof: The eigen-values of the characteristics equation $\lambda_{1}<0$ and $\lambda_{2}<0$ if $\mathrm{R}_{0}<1$ then the diseases free equilibrium $\mathrm{E}_{0}$ is locally asymptotically stable.

\subsection{Local Stability of Endemic Equilibrium point}

In this section, we discuss the local stability of endemic equilibrium of system (3) by analyzing the corresponding characteristic equations.

Theorem 4: $[26,27,32]$, The endemic equilibrium $E_{2}=\left(S_{2}, I_{2}\right)$ is locally asymptotically stable.

Proof: We begin by analyzing the stability of the endemic equilibrium point $\mathrm{E}_{2}$.

The Jacobean matrix of system (3) at the equilibrium point $\mathrm{E}_{2}$ is given by

$$
J \mid\left(S_{2}, I_{2}\right)=\left(\begin{array}{cc}
-\mu-\beta I_{2} & -\beta S_{2} \\
\beta I_{2} & \beta S_{2}-\mu-\gamma
\end{array}\right)
$$

Note that from $A-\mu S_{2}=\beta S_{2} I_{2}=(\mu+\gamma) I_{2}+r$, we have

$$
S_{2}=\frac{A-(\mu+\gamma) I_{2}-r}{\mu}
$$

Then trace of $\mathrm{J}_{2}$ is

$$
\begin{aligned}
& \operatorname{tr}\left(J_{2}\right)=-2 \mu-\beta I_{2}+\beta S_{2}-\gamma \\
& =-2 \mu-\beta I_{2}+\beta\left(\frac{A-(\mu+\gamma) I_{2}-r}{\mu}\right)-\gamma \\
& =\frac{-2 \mu^{2}-\mu \beta I_{2}+\beta A-\beta(\mu+\gamma) I_{2}-\beta r-\mu \gamma}{\mu} \\
& =-\left(\frac{2 \mu \beta+\gamma \beta}{\mu}\right) I_{2}-\frac{2 \mu^{2}-\beta A+\beta r+\gamma \mu}{\mu}
\end{aligned}
$$

Thus the trace is negative if $2 \mu^{2}-\beta A+\beta r+\gamma \mu \geq 0$

And then $\operatorname{det}\left(J_{2}\right)=\left(-\mu-\beta I_{2}\right)\left(\beta S_{2}-\mu-\gamma\right)+\beta^{2} S_{2} I_{2}$

$$
=-\beta A+2 \beta(\mu+\gamma) I_{2}+r \beta+\mu(\mu+\gamma)
$$




$$
\begin{aligned}
= & \mu(\mu+\gamma)\left[-R_{0}+\frac{2 \beta I_{2}}{\mu}+H+1\right] \\
= & \mu(\mu+\gamma)\left[-R_{0}+2 \frac{\beta}{\mu}\left(\frac{\mu}{2 \beta}\left(\mathrm{R}_{0}-1-H+\sqrt{\left(R_{0}-1-H\right)^{2}-4 H}+H+1\right)\right]\right. \\
= & \mu(\mu+\gamma)\left[-R_{0}+R_{0}+\sqrt{\left(R_{0}-1-H\right)^{2}-4 H}\right] \\
& \mu(\mu+\gamma)\left[\sqrt{\left(R_{0}-1-H\right)^{2}-4 H}\right]>0
\end{aligned}
$$

Since, $\operatorname{det}\left(J_{2}\right)=\mu(\mu+\gamma)\left[\sqrt{\left(R_{0}-1-H\right)^{2}-4 H}\right]>0$.

Therefore, $\operatorname{tr}\left(\mathrm{J}_{2}\right)<0$ and $\operatorname{det}\left(\mathrm{J}_{2}\right)>0$.

This implies that the eigen-value of Jacobean matrix $\mathrm{J}_{2}$ has negative real part, and hence $\mathrm{E}_{2}$ is locally asymptotically stable.

\subsection{Bifurcation Analysis}

The main purpose of this section is to get an insight into how the dynamics of the system changes depending on the system parameters. If a parameter is allowed to vary, the dynamics of the system may change. The stability of an equilibrium may change from an equilibrium point may appear or disappear a periodic solution may appear or disappear as the values of parameter varies. An equilibrium point may become unstable and a periodic solution may appear or a new stable equilibrium point may appear making the previous equilibrium point unstable. The change in the qualitative behavior of solution as a control parameter is varied is known as a bifurcation and the parameter values at which bifurcation occur are called bifurcation points.

An epidemic models, the reproduction number works as the threshold quantity for the stability of the diseasefree equilibrium. The usual situation is that for $\mathrm{R}_{0}<1$ the diseases-free equilibrium point is the only equilibrium and it is asymptotically stable, but it loses its stability as $\mathrm{R}_{0}$ increases through 1 , where a stable endemic equilibrium emerges, which depends continuously on $\mathrm{R}_{0}$. Such a transition of stability between the disease-free equilibrium point and the endemic equilibrium point is called forward bifurcation.

However, it is possible to have a very different situation at $\mathrm{R}_{0}=1$, as there might exist positive equilibria also for values of $R_{0}$ less than 1 . In this case we say that the system undergoes a backward bifurcation at $R_{0}=1$, when for values of $\mathrm{R}_{0}$ in an interval to the left of 1 , multiple positive equilibria coexist,( such as Disease free equilibrium and endemic equilbruim) typically one unstable and the other is stable.

When forward bifurcation occurs, the condition $\mathrm{R}_{0}<1$ is usually a necessary and sufficient condition for disease eradication, whereas it is no longer sufficient when a backward bifurcation occurs. In fact, the backward bifurcation scenario involves the existence of the trans-critical bifurcation at $\mathrm{R}_{0}=1$ and of a saddle-node bifurcation

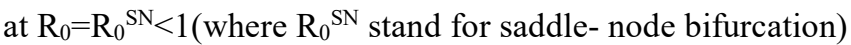

In particularly the backward bifurcation may be qualitatively described as follows. In the neighborhood of 1 , for $\mathrm{R}_{0}<1$, the stability of disease free equilibrium point is exists with two endemic equilibria: a smaller equilibrium (i.e., with a smaller number of infective individuals) which is unstable and a larger one (i.e., with a larger number of infective individuals) is stable. These two endemic equilibria disappear by saddle-node bifurcation when the basic reproductive number $\mathrm{R}_{0}$ is decreased below the critical value $\mathrm{R}_{0}=\mathrm{R}_{0}{ }^{\mathrm{SN}}>1$

For $\mathrm{R}_{0}>1$, there are only two equilibria: the disease free equilibrium point, which is unstable, and the larger endemic equilibrium, which is stable. As a consequence, in the backward bifurcation scenario, if $\mathrm{R}_{0}$ is nearly below unity, then the disease control strongly depends on the initial sizes of the various sub-populations. On the contrary, reducing $\mathrm{R}_{0}$ below the saddle-node bifurcation value $\mathrm{R}_{0}{ }^{\mathrm{SN}}$, may result in disease eradication, which is guaranteed provided that the disease free equilibrium is globally asymptotically stable. Hence, determining the sub-threshold $\mathrm{R}_{0} \mathrm{SN}$ may have a crucial importance in terms of disease control.

The linear stability analysis shows that diseases free equilibrium is locally asymptotically stable if $\beta \mathrm{A}<\mu(\mu+\gamma)$ and an unstable if $\beta \mathrm{A}>\mu(\mu+\gamma)$, while the endemic equilibrium points is locally asymptotically stable if $\beta \mathrm{A}>$ $\mu(\mu+\gamma)$ and an unstable if $\beta \mathrm{A}<\mu(\mu+\gamma)$. Furthermore, at $\beta \mathrm{A}=\mu(\mu+\gamma$, so there is a trans-critical bifurcation. There is the threshold condition $\mathrm{R}_{0}=1$ is equivalent to the threshold condition describes at $\mathrm{BA}=\mu(\mu+\gamma)$

When the disease free equilibrium point and the endemic equilibrium point exist and exchange stability, a unique stable endemic equilibrium point arises from the bifurcation point $R_{0}$ and increases as $R_{0}$ increases $(\beta$ increases by fixing $A$ and $\mu$ ). Thus, it shows that infectious free equilibrium exists for all $\mathrm{R}_{0}$, while endemic infections only exist for $\mathrm{R}_{0}>1$.

Let us verify that the existence of a Hopf bifurcation in (3) and determine its direction. 


$$
A_{0}=\frac{1}{2 \beta}\left[2 \beta A+(2 \mu+\gamma)(\mu+\gamma)\left(1-\sqrt{1+\frac{4 \beta A}{(\mu+\gamma)^{2}}}\right)\right]
$$

Theorem 4.5.1: [23] If case three is satisfied and

$$
\beta r<\beta A-3 \mu^{2}-\mu \gamma-2 \frac{\mu^{3}}{\gamma}
$$

then there is a family of unstable limit cycles if $r$ is less than and close to $A_{0}$ (that is subcritical Hopf bifurcation occurs when $r$ passes through the critical value $\mathrm{A}_{0}$ ).

Proof:

Let $\mathrm{r}=\mathrm{A}_{0}$. Then the $\operatorname{tr}\left(\mathrm{J}_{2}\right)=0$. It follows from (16) that

$$
\begin{aligned}
& \operatorname{tr}\left(J_{2}\right)=-2 \mu-\beta I_{2}+\beta S_{2}-\gamma=0 . \\
& \quad=-2 \mu-\beta I_{2}+\beta\left(\frac{A}{\mu+\beta I_{2}}\right)-\gamma
\end{aligned}
$$

Then we have

$$
\beta^{2} I_{2}^{2}-(3 \mu \beta+\gamma \beta) I_{2}+2 \mu^{2}-\beta A+\gamma \mu=0
$$

Therefore,

$$
\begin{aligned}
& I_{2}=\frac{1}{2 \beta}\left(\left((3 \mu+\gamma) \pm \sqrt{(3 \mu+\gamma)^{2}-4\left(2 \mu^{2}-\beta A+\gamma \mu\right)}\right)\right. \\
& S_{2}=\frac{\mu+\gamma}{2 \beta}\left(1+\sqrt{1+\frac{4 \beta A}{(\mu+\gamma)^{2}}}\right)
\end{aligned}
$$

From the above equations one can see that $\operatorname{tr}\left(\mathrm{J}_{2}\right)=0$ and $\operatorname{det}\left(\mathrm{J}_{2}\right)>0$.

Thus from the above conditions

Then, $\lambda_{1,2}= \pm \omega i$.

$$
\lambda_{1,2}= \pm i \sqrt{\mu(\mu+\gamma) \sqrt{\left(R_{0}-1-H\right)^{2}-4 H}}
$$

Now let $\omega=\sqrt{\mu(\mu+\gamma) \sqrt{\left(R_{0}-1-H\right)^{2}-4 H}}$

Perform coordinate transformation by $\mathrm{x}=\mathrm{S}-\mathrm{S}_{2}$ and $\mathrm{y}=\mathrm{I}-\mathrm{I}_{2}$ then system (3) will become

$$
\left\{\begin{array}{l}
\frac{d x}{d t}=-\left(\mu+\beta I_{2}\right) x-\beta S_{2} y-\beta x y \\
\frac{d y}{d t}=\beta I_{2} x+\left(\beta S_{2}-(\mu+\gamma)\right) y+\beta x y
\end{array}\right.
$$

Setting

and from

$$
x=-\beta S_{2} V, y=\omega U+(\mu+\beta) V
$$

$$
\begin{gathered}
\operatorname{tr}\left(\mathrm{J}_{2}\right)=-2 \mu-\beta \mathrm{I}_{2}+\beta \mathrm{S}_{2}-\gamma=0 \text { and } \\
\omega^{2}=\operatorname{det}\left(J_{2}\right)=-\mu \beta \mathrm{S}_{2}+\mu^{2}+\gamma \mu+\beta \mu I_{2}
\end{gathered}
$$

we obtain

$$
\left\{\begin{array}{l}
\frac{d U}{d t}=-\omega V+F(U, V), \\
\frac{d V}{d t}=\omega U+G(U, V)
\end{array}\right.
$$


where $F(U, V)=\frac{\beta V\left(-\beta S_{2}+\beta I_{2}+\mu\right)\left(\omega U+\mu V+\beta I_{2} V\right)}{\omega}$

$$
G(U, V)=-\beta V\left(\omega U+\mu V+\beta I_{2} V\right)
$$

We know that $\operatorname{tr}\left(\mathrm{J}_{2}\right)=0$ and hence we get

$$
G(U, V)=\frac{\omega F(U, V)}{\mu+\gamma}
$$

And we have the following:

$$
\begin{aligned}
& F_{U}=\beta V\left(-\beta S_{2}+\beta I_{2}+\mu\right), F_{U U}=0, F_{U U U}=0, F_{U V}=\beta\left(-\beta S_{2}+\beta I_{2}+\mu\right), F_{U V V}=0, \\
& F_{V}=\frac{\omega U \beta\left(-\beta S_{2}+\beta I_{2}+\mu\right)+2 \mu \beta V\left(-\beta S_{2}+\beta I_{2}+\mu\right)+2 \beta^{2} \mu I_{2} V\left(-\beta S_{2}+\beta I_{2}+\mu\right)}{\omega} \\
& F_{V V}=\frac{2 \mu \beta\left(-\beta S_{2}+\beta I_{2}+\mu\right)+2 \mu \beta^{2} I_{2}\left(-\beta S_{2}+\beta I_{2}+\mu\right)}{\omega} \\
& G_{U}=\frac{\beta V\left(-\beta S_{2}+\beta I_{2}+\mu\right) \omega}{\mu+\gamma}, G_{U V}=\frac{\beta\left(-\beta S_{2}+\beta I_{2}+\mu\right) \omega}{\mu+\gamma}, G_{U U}=0, G_{U U V}=0, \\
& G_{V}=\frac{\omega \beta U\left(-\beta S_{2}+\beta I_{2}+\mu\right)+2 V \beta\left(-\beta S_{2}+\beta I_{2}+\mu\right)+2 \beta^{2} V I_{2}\left(-\beta S_{2}+\beta I_{2}+\mu\right)}{\mu+\gamma}, \\
& G_{V V}=\frac{2 \mu \beta\left(-\beta S_{2}+\beta I_{2}+\mu\right)+2 \beta^{2} I_{2}\left(-\beta S_{2}+\beta I_{2}+\mu\right)}{\mu}, G_{V V V}=0
\end{aligned}
$$

Substituting these in the relation

$$
\begin{aligned}
& \mu=\frac{1}{16}\left[F_{U U U}+F_{U V V}+G_{U U V}+G_{V V V}\right]+\frac{1}{16 \omega}\left[F_{U V}\left(F_{U U}+F_{V V}\right)-G_{U V}\left(G_{U U}+G_{U V}\right)-F_{U U} G_{U U}+F_{V V} G_{V V}\right. \\
& \mu=\frac{1}{16 \omega}\left[\beta\left(-\beta S_{2}+\beta I_{2}+\mu\right)\left(\frac{2 \mu \beta\left(-\beta S_{2}+\beta I_{2}+\mu\right)+2 \mu \beta^{2} I_{2}\left(-\beta S_{2}+\beta I_{2}+\mu\right)}{\omega}\right)\right. \\
& -\frac{\beta\left(-\beta S_{2}+\beta I_{2}+\mu\right) \omega}{\mu+\gamma}\left(\frac{2 \mu \beta\left(-\beta S_{2}+\beta I_{2}+\mu\right)+2 \beta^{2} I_{2}\left(-\beta S_{2}+\beta I_{2}+\mu\right)}{\mu+\gamma}\right) \\
& \left.+\left(\frac{2 \mu \beta\left(-\beta S_{2}+\beta I_{2}+\mu\right)+2 \mu \beta^{2} I_{2}\left(-\beta S_{2}+\beta I_{2}+\mu\right)}{\omega}\right) \frac{2 \mu \beta\left(-\beta S_{2}+\beta I_{2}+\mu\right)+2 \beta^{2} I_{2}\left(-\beta S_{2}+\beta I_{2}+\mu\right)}{\mu+\gamma}\right]
\end{aligned}
$$

Then after some algebraic calculations we obtain

$$
\mu=-\frac{\left.\beta^{2}\left(-\beta S_{2}+\beta I_{2}+\mu\right)^{2}\left(\mu+\beta I_{2}\right)\left(-3 \mu^{2}-4 \gamma \mu-\gamma^{2}\right)+\omega^{2}-2 \mu \beta I_{2}-2 \mu \gamma I_{2}\right)}{8(\mu+\gamma)^{2}}
$$

Since $\operatorname{tr}\left(\mathrm{J}_{2}\right)=-2 \mu-\beta \mathrm{I}_{2}+\beta \mathrm{S}_{2}-\gamma=0=-\beta \mathrm{S}_{2}+\beta \mathrm{I}_{2}+\mu=-\mu-\gamma$.

we have

$$
\mu=\frac{\beta^{2}\left(\mu+\beta I_{2}\right)\left(2 \mu^{2}+3 \gamma \mu+\gamma^{2}+2 \beta S_{2}+\gamma \beta I_{2}\right)}{8 \omega^{2}}>0
$$

Then the conclusion of this theorem follows from L. Perko [12]

As an example, we fix $\mathrm{A}=8, \mu=0.1, \beta=1, \gamma=1$. Then we obtain 


$$
\left(\sqrt{R_{0}}-1\right)^{2} \mu(\mu+\gamma)=6.2338, \beta A-3 \mu^{2}-d \gamma-\frac{2 \mu^{3}}{\gamma}=7.868 \quad \text { and } \mathrm{A}_{0}=5.2023 \text {. }
$$

We know that there is an unstable limit cycle when $\mathrm{r}$ is less than and near $\mathrm{A}_{0}$ from theorem 4.5.1, which is shown that there is an unstable limit cycle when $r$ decrease from 5.2023

\section{THE NUMERICAL SIMULATION}

In this section we give the numerical simulation of system (3).

We take the parameters of the system as $\mathrm{A}=4.7, \mu=4.29, \beta=4.1, \gamma=0.69, \mathrm{r}=0.98$. Then $\mathrm{E}_{0}=(1.09,0,0)$. Therefore $\mathrm{E}_{0}$ is a global stable.

For the removal rate $(r=0.87), E_{1}=(7.95,0.67)$ and $E_{2}=(6.14,1.16)$, equilibrium $E_{2}$ will remain unstable and the disease will become extinct in finite time. For a lower removal rate $(r=0.6), E_{1}=(10.21,0.30) ; E_{2}=(4.78$, 1.78), equilibrium $E_{2}$ will become locally asymptotically stable and the disease will become persistence in finite time.

For the initial value, the approximated solutions $S(t), I(t)$, and $R(t)$ are displayed in the figure (6.1-6.4) given below.

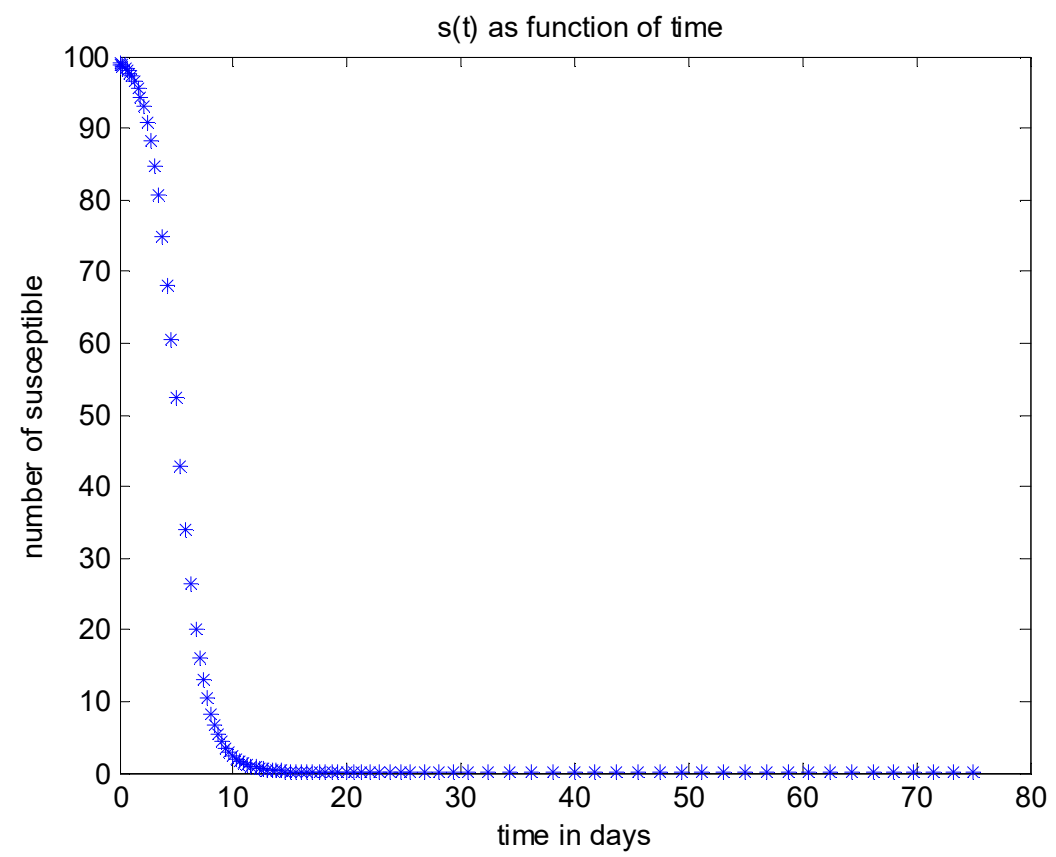

Figure 6.1: Simulation of Susceptible Population

Figure 6.1 is the diagram showing the dynamics of the susceptible population. The Susceptible population decreases as time increases. This decrease may be possibly because of the high rate of recovery due to mass vaccination, since individual become permanently immune upon recovery. The contact rate also has large impact on the spread of a disease through a population. The higher the rates of contact, the more rapid the spread of the disease, it is also observed that as the contact rate decreases, the fraction of individuals infected decreases at a faster rate as would be expected logically. 


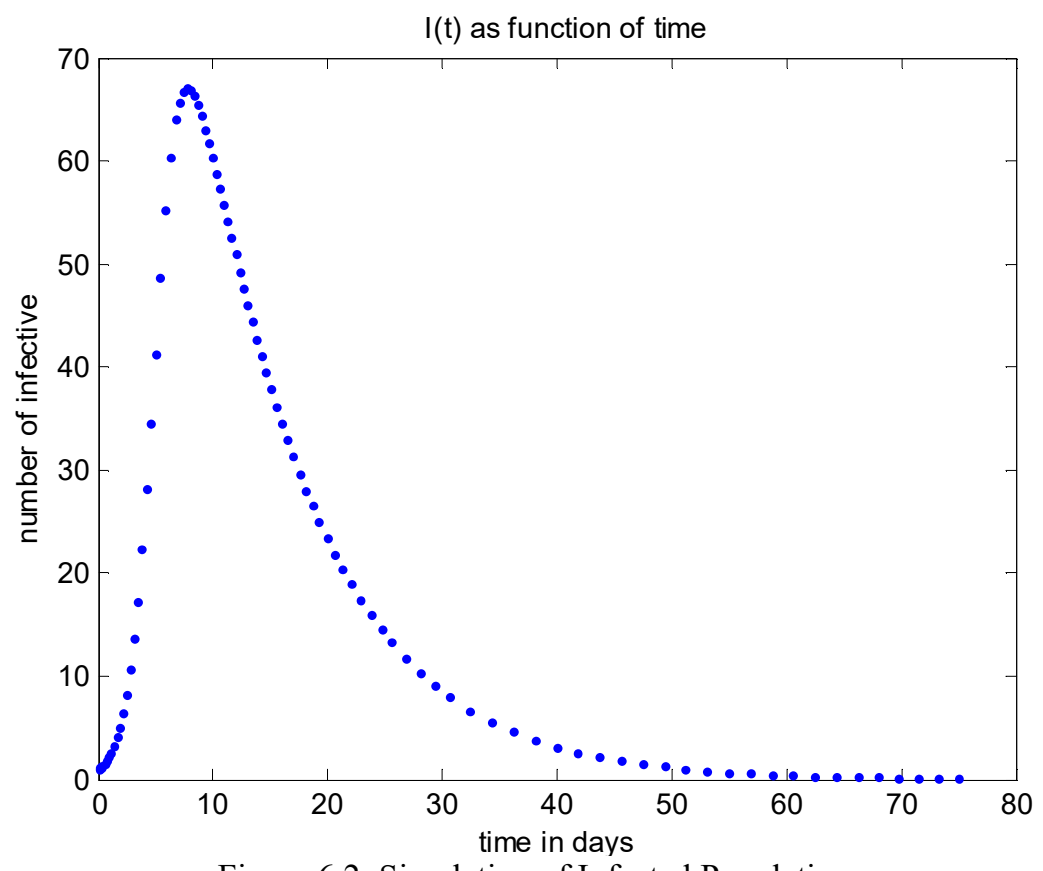

Figure 6.2: Simulation of Infected Population.

Observing Figure 6.2, The simulation shows that $\mathrm{I}(\mathrm{t})$ is initially increasing as the rate increases, then after reaching a maximum then it is decreasing. This rapid decline of the infected individuals may be due to early detection of the disease. If we determine the possible maximum points of $I(t)$ by examining the equation $I^{\prime}(t)=0$, $S(t)=\frac{\mu+\gamma}{\beta}$ maximum when $S(t)=\frac{\mu+\gamma}{\beta}$ and $\mathrm{I}(\mathrm{t})$ can attain its maximum at most once. Also $\mathrm{I}(\mathrm{t})$ is increasing $\left(\mathrm{I}^{\prime}(\mathrm{t})>0\right)$ $S(t)>\frac{\mu+\gamma}{\beta}$.This show that an epidemic.

And $\mathrm{I}(\mathrm{t})$ decreasing $\left(\mathrm{I}^{\prime}(\mathrm{t})<0\right)$ when $\quad$ w $(t)<\frac{\mu+\gamma}{\beta}$. This show that the disease is dies out. The condition $S(0)>\frac{\mu+\gamma}{\beta}$ decreasing from the very beginning. This graph also demonstrates that the contact rate has large impact on the spread of the disease through population. If the contact rate is observed to be high then the rate of infection of the disease will also be high as would be expected logically. However, there exists another parameter to consider, as more individuals are infected with the disease and $\mathrm{I}(\mathrm{t})$ grows, some individuals are also leaving the infected class by being cured and then join the Recovered class. 


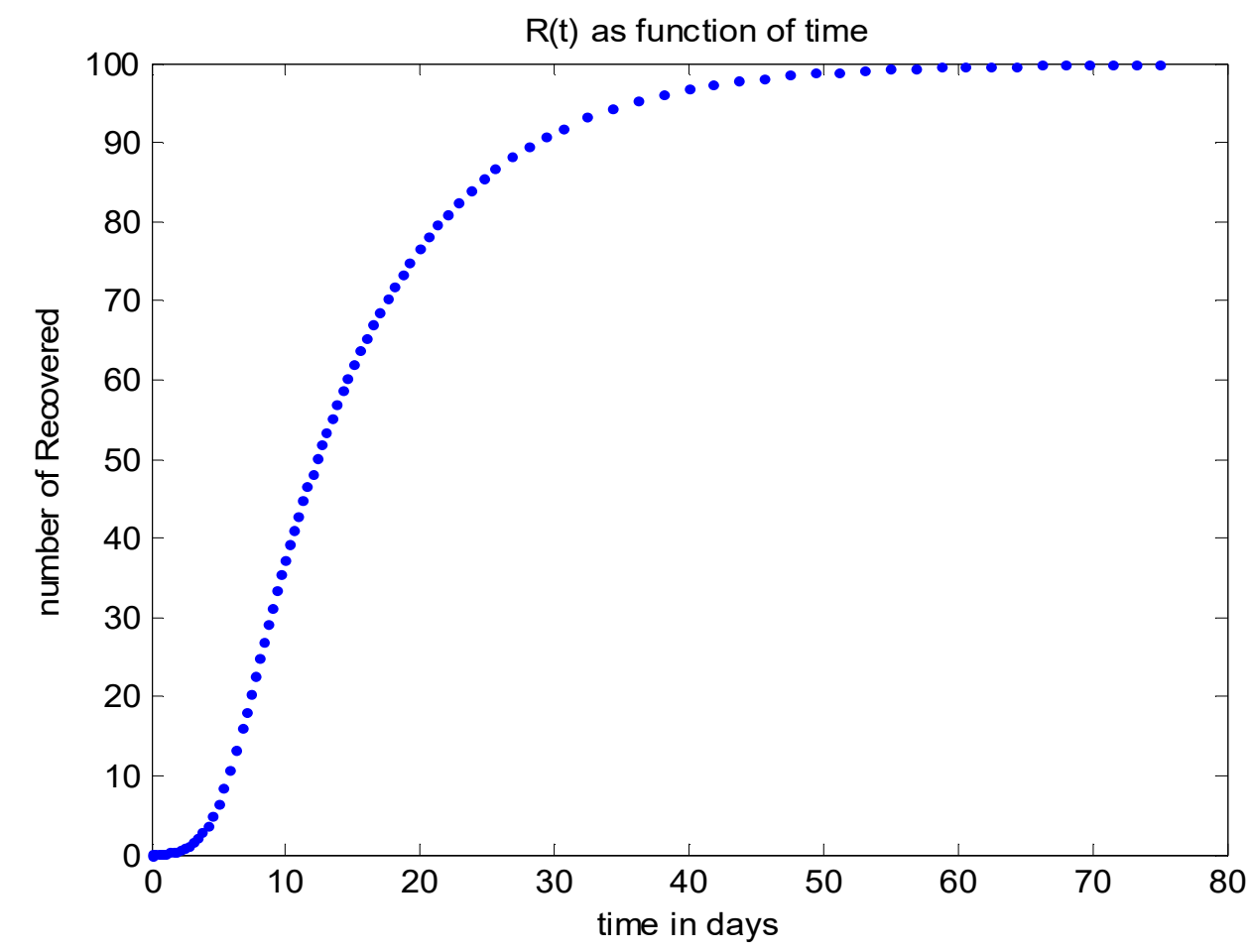

Figure 6.3: Simulation of Recovered Population.

In figure 6.3 the number of individuals Recovered is rise steadily as rate increases. This may be due to early detection of the disease as well as education about the diseases transmission. It can also be observed that the population of the recovered individuals rise up steadily for some number of years and then drops and remains nearly a constant. This could be due to the greater number of infectious individuals who have been treated and also acquired education about the diseases transmission.

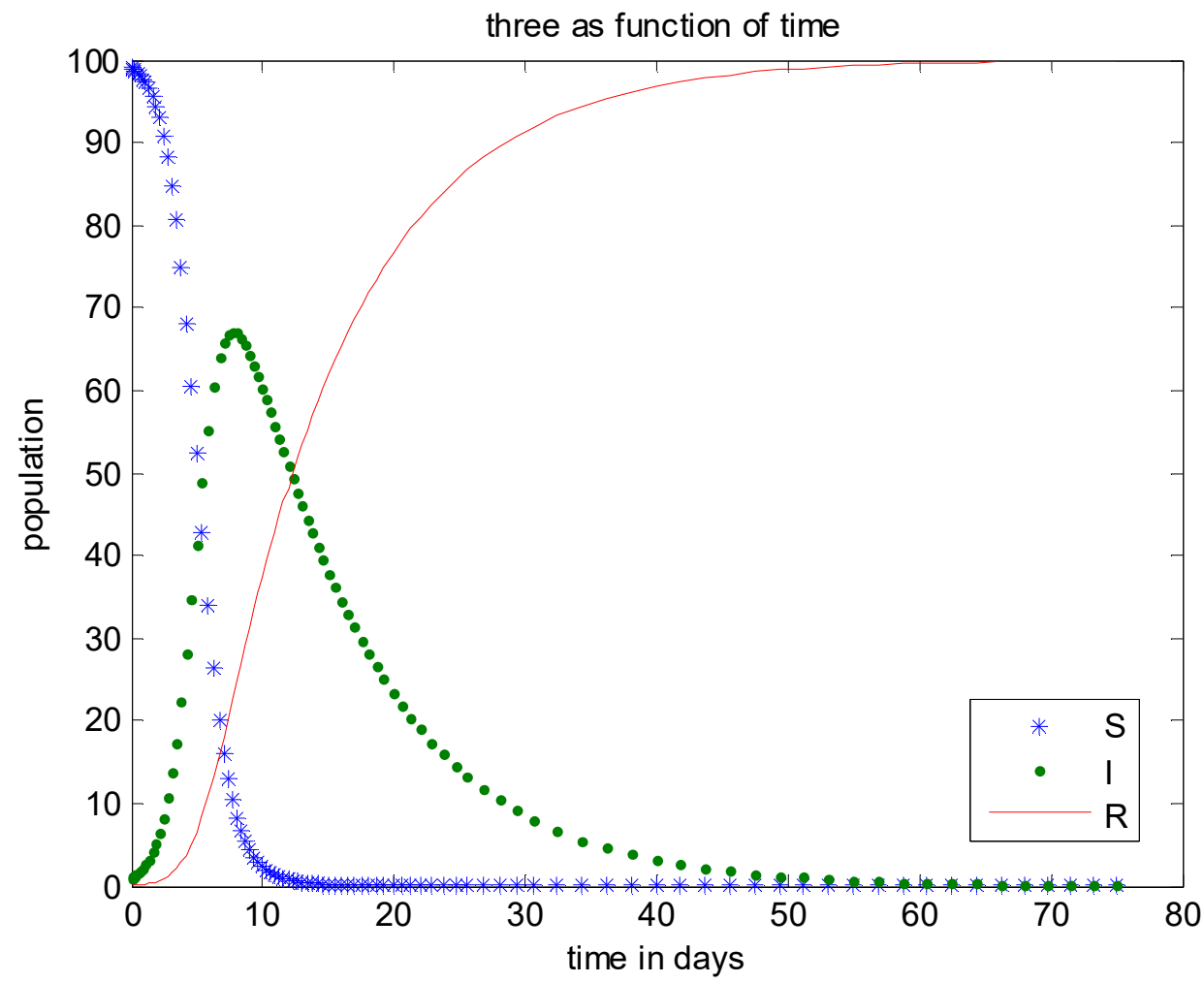

Figure.6.4: The three populations versus time with $\mathrm{A}=4.1, \mu=0.29, \beta=4.7, \gamma=0.69, \mathrm{r}=0.98$

Fig. 6.4 shows that $S(t)$ approaches to its equilibrium value while $I(t)$ and $R(t)$ approach zero as time 
progresses, the disease dies out.

\section{CONCLUSION}

In this thesis, by combining qualitative and bifurcation analyses we have studied the global behavior of an epidemic model with a constant removal rate of the infective individuals to understand the effect of the treatment capacity on the disease transmission. We have shown that there are two possibilities for the outcome of the disease transmission. First, if $\mathrm{R}_{0}<1$ there is a disease -free equilibrium which is asymptotically stable and the infections dies-out. Second, if $\mathrm{R}_{0}>1$ the usual situation is there is an endemic equilibrium which is asymptotically stable and the infections persist. If the endemic equilibrium is unstable the instability commonly arises from a Hopf bifurcation and the infection still persists.

More precisely, as $\mathrm{R}_{0}$ is increase through 1 there is an exchange of stability between the disease -free equilibrium and the endemic equilibrium (which is negative as well as unstable and the biologically meaningless if $\mathrm{R}_{0}<1$. There is bifurcation or change in equilibrium behavior at $\mathrm{R}_{0}=1$ but the equilibrium infective population size depends on continuously on $\mathrm{R}_{0}$ such a transition is a transcritional bifurcation. In this case, reducing the basic reproductive number $\mathrm{R}_{0}$ below one may fail to control the disease.

Generally the model has shown success in attempting to predict the causes of diseases transmission within a population. The model strongly indicated that the spread of a disease largely depend on the contact rates with infected individuals within a population

\section{References:}

[1] R.M. Anderson, R.M. May, Infectious Diseases of Humans, Dynamics and Control, Oxford University Press, Oxford, 1991.

[2] R. Bogdanov, Bifurcations of a limit cycle for a family of vector fields on the plan, Selecta Math. Soviet. 1 (1981) 373-388

[3] R. Bogdanov, Versal deformations of a singular point on the plan in the case of zero eigen-values, Selecta Math. Soviet. 1 (1981) 389-421.

[4] F. Brauer, P. van den Driessche, Models for the transmission of disease with immigration of infectives, Math. Biosci. 171 (2001) 143-154.

[5] O. Diekmann, M. Kreztschmar, Patterns in the effects of infectious disease on population growth, J. Math. Biol. 29 (1991) 539-570.

[6] D.J.D. Earn, P. Rohani, B.M. Bolker, B.T. Grenfell, A simple model for complex dynamical transitions inepidemics, Science 287 (2000) 667-670.

[7] H.W. Hethcote, S.A. Levin, Periodicity in epidemiological models, in: S.A. Levin, T.G. Hallam, L.J. Gross (Eds.), Applied Mathematical Biology, in: Biomath. Texts, vol. 18, Springer-Verlag, New York, 1989, pp. 193-211.

[8] H.W. Hethcote, H.W. Stech, P. van den Driessche, Nonlinear oscillations in epidemic models, SIAM J. Appl. Math. 40 (1981) 1-9.

[9] Z. Feng, H.R. Thieme, Recurrent outbreaks of childhood diseases revisited: the impact of isolation, Math. Biosci. 128 (1995) 93-130.

[10] L. Wu, Z. Feng, Homoclinic bifurcation in an SIQR model for childhood diseases, J. Differential Equations 168 (2000) 150-167.

[11] J.M. Hyman, J. Li, Modeling the effectiveness of isolation strategies in preventing STD epidemics, SIAM J.Appl. Math. 58 (1998) 912-925.

[12] Perko, L. Differential Equations and Dynamical Systems. Springer-Verlag, New York 1996

[13] W.O. Kermack, A.G. McKendrick, A contribution to the mathematical theory of epidemics,Proceedings of the Royal society of London. Series A, 115, no. 772, 700-721 (1927)

[14] V. Capasso and G. Serio, "A generalization of the KermackMcKendrick deterministic epidemic model,"Mathematical Biosciences, vol.42,no.1-2,pp.43-61,1978.

[15] Hethcote H.W, Tudor D.W (1980). Integral equation models for epidemic infectious diseases, J. Math. Biol, 9, pp.37-47.

[16] W.M. Liu, H.W. Hethcote, S.A. Levin, Dynamical behavior of epidemiological models with nonlinear incidence rates, J. Math. Biol. 25 (1987) 359-380.

[17] Hethcote H.W, Driessche P.van den (1991). Some Epidemiological model with nonlinear incidence,J. Math.Biol, 29, pp. 271-287.

[18] Derrick W.R, Driessche P. van den (1993). A disease transmission model in non-constant population, J. Math. Biol. 31, pp. 495-512.

[19] Beretta E, Takeuchi Y (1995). Global stability of a SIR epidemic model with time delay, J. Math. Biol. 33, pp. 250-260.

[20] Beretta E, Takeuchi Y (1997). Convergence results in SIR epidemic model with varying population sizes, 
Nonlinear Anal. 28, pp. 1909-1921.

[21] Beretta E, Hara T, Ma W, Takeuchi Y (2001). Global asymptotic stability of a SIR epidemic model with distributed time delay, Nonlinear Anal. 47, pp. 4107-4115.

[22] Ma W, Hara T, Beretta E, Takeuchi Y (2002). Permanence of an SIR epidemic model with distributed time delays, Tohoku Math. J. 54, pp. 581-591.

[23] Runa S, Wang W (2003). Dynamical behavior of an epidemic model with nonlinear incidence rate, J. Differ. Equations, 188, pp. 135-163.

[24] Ma W, Song M, Takeuchi Y (2004). Global stability of an SIR epidemic model with time delay, Appl. Math.Lett. 17, pp.1141-1145.

[25] Xiao D, Runa S (2007). Global analysis of an epidemic model with non-monotone incidence rate, Math. Biosci. 208, pp. 419-429.

[26] S. Ruan, W. Wang, Dynamical behavior of an epidemic model with a nonlinear incidence rate, J. Differential Equations 188 (2003) 135-163 